\title{
SEVERIDAD DE Peronospora variabilis GÄUM. EN Chenopodium quinoa WILLD. 'PASANKALLA' COMO RESPUESTA A APLICACIONES DE FUNGICIDAS SINTÉTICOS Y BIOESTIMULANTES
}

\author{
EFFECT OF FUNGICIDES AND BIOSTIMULANTS ON THE SEVERITY OF QUINOA \\ DOWNY MILDEW.
}

${ }^{1}$ Alejandro Risco M. y ${ }^{1}$ Leonor Mattos C.

\begin{abstract}
Resumen
A nivel nacional el cultivo de quinua ha adquirido mucha importancia para la agro exportación, pero el "Mildiú" causado por Peronospora variabilis ocasiona grandes pérdidas. Por ello, se planteó como objetivo determinar el efecto de las aplicaciones foliares de productos con acción fungicida en el progreso del mildiú en quinua variedad Pasankalla. Los tratamientos fueron: Fosfito de potasio (FP), Azúcar fosfatado (KO), Lactobacillus (E), Fermentados de sólidos soluble (A), FP+E, KO+E, FP+A, KO+A, Metalaxyl (TM) y Testigo absoluto sin aplicación (T). Se evaluó: Área Bajo la Curva del Progreso de la Enfermedad (ABCPE), tasa del progreso del mildiú ( $r$ ), número de oosporas/kg de semilla y rendimiento. La severidad de la enfermedad se evaluó cada dos días haciendo un total de 20 evaluaciones. El valor más alto del ABCPE lo tuvo el testigo absoluto $(442,43)$; los valores más bajos fueron obtenidos en Metalaxyl $(175,43)$ y el Fosfito de potasio $(199,96)$. Las $r$ más bajas fueron registradas en los tratamientos FP+E $(0,015)$, Fosfito de potasio $(0,017)$ y Metalaxyl $(0,017)$. El menor número de oosporas en semillas se registró en los tratamientos Metalaxyl y FP+A (3500 oosporas $/ \mathrm{kg}$ ); mientras que, el Testigo obtuvo el mayor número con 17500 oosporas $/ \mathrm{kg}$. Los mejores rendimientos se obtuvieron con los tratamientos Fosfito de potasio, Metalaxyl y FP+E $(4059,3686,3568 \mathrm{~kg} /$ ha) respectivamente. Los tratamientos fosfito de potasio y Metalaxyl registraron mayores ingresos con respecto al testigo absoluto. En conclusión, estos resultados demuestran que el tratamiento a base de fosfito de potasio es una alternativa para el manejo del mildiu y para mejorar la sanidad del grano de quinua.
\end{abstract}

Palabras clave: fosfito de potasio, metalaxyl, mildiú.

\begin{abstract}
Quinoa (Chenopodium quinoa) is an important Andean crop has become very important crop for the export agriculture and downy mildew caused by Peronospora variabilis induce severe economic yield losses each year. The main objetive of this study was to test the effect of foliar applications of products with fungicidal action in the control quinua downy mildew. The treatments included: Potassium phosphite (FP), Sugar phosphate (KO), Lactobacillus (E), Fermented soluble solids (A), FP + E, KO + E + A FP, KO + A, metalaxyl 35\% (TM) and Control non-treated (T). The variables evaluated included: The area under the disease progression curve (AUDPC), disease Progression rates $(r)$, number of oospores by one kilogram of quinoa seed and grain yield. The mildew severity on plants was evaluated every two days for a total of 20 evaluations. The Control showed the highest AUDPC value $(442,43)$, whereas the metalaxyl and potassium phosphite treatments showed the lowest AUDPC values 175,43 and 199,96, respectively. Additionally, the lowest rates $(r)$ was observed in the FP + E $(0,015)$, potassium phosphite $(0,017)$ and metalaxyl $(0,017)$ treatments. The lowest number of oospores was recovered from quinoa seeds after treated with the $\mathrm{FP}+\mathrm{A}$ and metalaxyl treatments (3500 oospores $/ \mathrm{kg}$ ), whereas the highest number of oospores was recovered from the Control seeds (17500 oospores/ $\mathrm{kg}$ ). The best grain yield responses to chemical treatments were potassium phosphite, metalaxyl and FP + E treatments $4059,3686,3568 \mathrm{~kg} / \mathrm{ha}$, respectively. Potassium phosphite and metalaxyl treatments recorded the highest net return compared to the Control. In conclusion, these results showed that treatment with potassium phosphite is an alternative for management of mildew and to improve the health of quinoa grain.
\end{abstract}

Key words: metalaxyl, mildew, potassium phosphite.

\section{Introducción}

La quinua es un grano nativo originario de América del Sur, de las áreas andinas de Perú y Bolivia que es cultivado desde hace 7000 años y que en la actualidad se siembra en muchos países del mundo por su importancia nutricional en proteínas, aceites, minerales, fibras, etc. También es importante por su ausencia del gluten que hace apta para aquellos pacientes celiacos. Su producción 
es también importante para la seguridad alimentaria y la economía de las comunidades campesinas del Altiplano peruano-boliviano y valles interandinos de Perú, Ecuador y Colombia.

La producción de quinua en el Perú se incrementó de 28668 hectáreas en el 2003 a 56605 al 2014 (MINAGRI 2014). Así mismo, se reporta que la siembra se da en 14 regiones del Perú, donde Puno representa el 59 por ciento del área cultivada seguido de Ayacucho con el 12,5 por ciento; sin embargo, Arequipa y Lambayeque tienen los mejores rendimientos con 3979,5 y $2931,3 \mathrm{~kg} /$ ha respectivamente.

La enfermedad más importante de la quinua es el mildiú, causado por Peronospora variabilis Gäum., que ocasiona pérdidas en el rendimiento entre 33 y 58 por ciento en condiciones de alta presión de inóculo, pudiendo llegar hasta un 100 por ciento cuando las condiciones son favorables para su desarrollo: Humedad relativa mayor a 80 por ciento y temperatura entre 15 y $25^{\circ} \mathrm{C}$.

Por la importancia del mildiú en el cultivo de quinua, en esta investigación se probó productos de origen natural y químico aplicados a las hojas, con el fin de controlar a la enfermedad y se planteó como objetivo: determinar el efecto de las aplicaciones foliares de productos con acción fungicida en el progreso de la enfermedad causada por Peronospora variabilis Gäum. en Chenopodium quinoa Willd. 'Pasankalla'.

\section{Materiales y métodos}

Ubicación de la investigación: la investigación se realizó en el campo experimental del Programa de Cereales y Granos Nativos, y en el laboratorio de Fitopatología de la Universidad Nacional Agraria La Molina, ubicado en la región Lima a una altitud de 243 m.s.n.m. Con temperaturas que oscilaron entre 15 y $17{ }^{\circ} \mathrm{C}$; humedad relativa entre 85 y $95 \%$.

Material genético y productos para el control de mildiú: se empleó semilla de la variedad Pasankalla, y las propuestas para el control del mildiú fueron: Fosfito de potasio $70 \%(700 \mathrm{~g} / \mathrm{L})$ equivale a $475 \mathrm{~g} / \mathrm{L}$ de ácido fosforoso (Kalex), producto compuesto de la fermentación de sólidos solubles rico en nutrientes y aminoácidos (AGRO MOS), bioestimulante vegetal y antagonista de los complejos enzimáticos celulares (Kolapzer), consorcio de microorganismos benéficos principalmente bacterias lácticas Lactobacillus sp. (Ecovida), metalaxyl (Fitoklin) y testigo absoluto sin aplicación (Tabla 1).

Dosis y frecuencia de aplicación: los tratamientos con Fosfito de potasio $\left(500 \mathrm{cc} / \mathrm{cil}^{2}\right)$, Azúcares fosfatados (4 L/ha) y Metalaxyl (200 g/cil) se aplicaron en tres oportunidades a partir de los 24 días después de la siembra (dds) con una frecuencia de 15 días. Los tratamientos con Lactobacillus (1000 cc/cil) y Fermentados de sólidos solubles $(500 \mathrm{cc} / \mathrm{cil})$ se aplicaron en seis oportunidades,

${ }^{2}$ Cilindro: contenedor con capacidad de 200 litros. a partir de los 12 dds con una frecuencia de 10 días haciendo un total de seis aplicaciones.

Tabla 1. Tratamientos para el control de $P$. variabilis en quinua Pasankalla. La Molina 2013.

\begin{tabular}{clc}
\hline N$^{\circ}$ & Código & Tratamientos \\
\hline 1 & FP & Fosfito de potasio (Kalex) \\
2 & KO & Azúcar fosfatado (Kolapzer) \\
3 & E & Lactobacillus (Ecovida) \\
4 & A & Fermentados de sólidos solubles \\
5 & FP+E & Fosfito de potasio + Lactobacillus \\
6 & KO+E & Azúcar fosfatado + Lactobacillus \\
7 & FP+A & Fosfito de potasio + Fermentados de \\
8 & KO+A & Azúcar fosflidos solubles \\
9 & TM & sólidos solubles \\
10 & T & Testigo Metalaxyl (Fitoklin) \\
\hline
\end{tabular}

Diseño experimental: el campo experimental estuvo constituido de tres bloques, cada uno conformado por 10 parcelas de cuatro surcos de $4 \mathrm{~m}$ de largo y $0,8 \mathrm{~m}$ de distanciamiento entre surcos. Se utilizó el diseño de bloques completos al azar (DBCA), con comparaciones de medias Tukey y un nivel de significación de 0,05.

Siembra, fertilización y labores agronómicas: la siembra se realizó el 02 de agosto y la cosecha el 11 de diciembre con 130 días después de la siembra. Se abonó con úrea, fosfato diamónico y cloruro de potasio con niveles de 100 - 80 - 20 de $\mathrm{N}$ - $\mathrm{P}_{2} \mathrm{O}_{5}-\mathrm{K}_{2} \mathrm{O}$; materia orgánica a dosis de tres $\mathrm{t} / \mathrm{ha}$. Las labores culturales consistieron en una primera remoción de suelo o cultivado a los 11 dds y un segundo centrado o aporque definitivo a los $25 \mathrm{dds}$.

Evaluación sanitaria: en los dos surcos centrales de cada parcela se marcaron 20 plantas para evaluar severidad de la enfermedad cada dos días, haciendo un total de 20 evaluaciones. La intensidad de la severidad (\%) se determinó por aproximación o estimación visual directa de la proporción o porcentaje afectada de una planta completa, para lo cual de empleó la variable cuantitativa discretas que osciló entre 0 y 100 porciento.

Variables evaluadas: con los datos de severidad se calcularon las áreas bajo las curvas de progreso de la enfermedad (ABCPE), descrito originalmente en inglés como 'area under disease progress curve' (AUDPC) (Vanderplank, 1963; Shaner y Finney, 1977).

$$
\mathrm{AUDPC}=\sum_{i}^{n-1}\left[\frac{y_{i}+y_{i+1}}{2}\right]\left(\mathrm{t}_{i+1}+\mathrm{t}_{i}\right)
$$

Donde $n$ es el número de evaluaciones, $y$ es la severidad y $t$ es el número de días después de la siembra en la cual que se hace la evaluación. Se incluye $(t, y)=(0,0)$ como la primera evaluación.

La tasa $(r)$ del progreso de la enfermedad de las diez 
epidemias o tratamientos se ajustaron a distintos modelos epidemiológicos (Exponencial, Logístico y Gompertz). Por ello, se estandarizaron a la tasa relativa del incremento de la enfermedad $\rho$ (rho) para ser comparados estadísticamente (Madden 1986).

$$
\rho=\frac{\mathbf{r} * \mathbf{k}}{2 \mathbf{m}+2}
$$

Dónde $r$ es la tasa de la enfermedad, $k$ nivel máximo teórico de la intensidad de la enfermedad $(0 \leq \mathrm{k} \geq 1)$, $m$ parámetro forma, $(m=2,0,1$ ó 2 para los modelos Exponencial, Monomolecular, Gompertz y Logístico, respectivamente).

Para el análisis en laboratorio, se extrajeron muestras de hojas con síntomas de mildiú y se hicieron observaciones de: esporulación en el envés de las hojas usando un microscopio estereoscopio a magnificación de 100X, esporangióforos y esporangios con un microscopio binocular a una magnificación de 400X.

El conteo de oosporas $\left(\mathrm{N}^{\circ} / \mathrm{kg}\right)$, se efectuó mediante el método combinado y modificado de Danielsen (2008), para Peronospora variabilis en quinua, y Pathank (1978), para Peronospora manshurica en soya. El método consistió en pesar un gramo de quinua para remojarlos en agua destilada durante 60 minutos, cada 10 minutos se agitó por un periodo de tres minutos. Se tomó cinco mililitros de la suspensión y se centrifugó durante seis minutos a velocidad 9 (aprox. $2500 \mathrm{rpm}$ ) en una centrifuga Germmy PCL-05. El sobrenadante se eliminó $\mathrm{y}$ el sedimento fue resuspendido en seis gotas de agua destilada. Finalmente, dos gotas de la suspensión fueron examinadas con un microscopio a 100X de magnificación.

Para la calidad de grano, se consideraron las siguientes variables: contenido de proteína (\%), se midió usando el método físico, con el equipo Infratec ${ }^{\mathrm{TM}} 1241$ Grain Analyser; el peso de 1000 granos (g), empleando el equipo COUNT-A-PAK ${ }^{\circledR}$ SEED que a través de un sensor controlado por un microprocesador las semillas fueron detectados para contabilizarlas y posteriormente pesarlas; granulometría (\%), se pesaron $20 \mathrm{~g}$ de granos para seleccionarlas por diferentes tamaños con una zaranda vibratorias de calibres $2 ; 1,7$ y 1,4 mm, se agitó por un periodo de cinco minutos $\mathrm{y}$, finalmente, fueron pesados para calcular el porcentaje de cada calibre.

\section{Resultados y discusión}

En el envés de las hojas de quinua Pasankalla se observaron signos de $P$. variabilis, la esporulación fue de color plomizo visto al estereoscopio (Fig. 1-A); zoosporangióforos y zoosporangios vistos al microscopio a 400X (Fig. 1-B) y sobre el pericarpio de las semillas, las oosporas, con coloración anaranjada, forma esférica, de pared delgada, clara y ondulada en estado joven; pared gruesa, oscura y redondeado en estado maduro (Fig. 2) (Danielsen y Ames 2008).

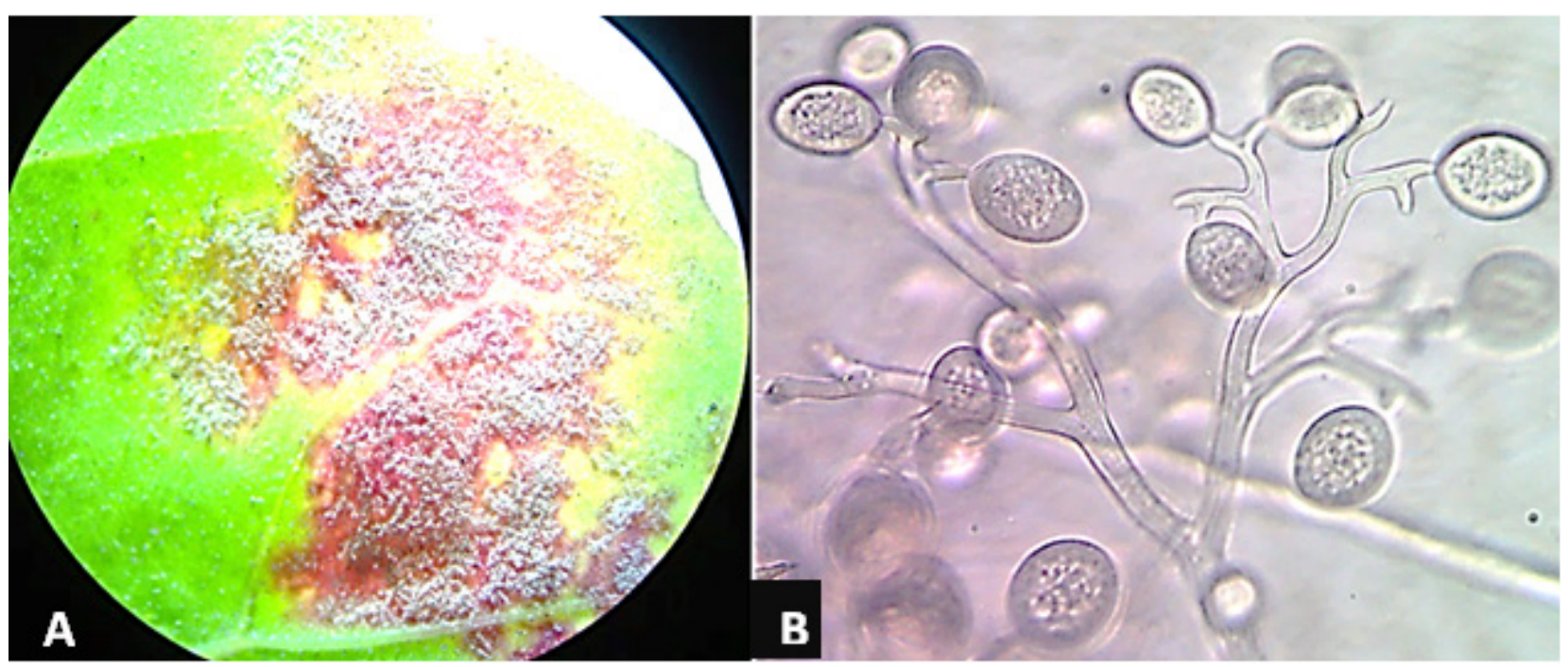

Figura 1. A. Envés de la hoja de quinua con signo de esporulación plomiza; B. Zoosporangióforo y zoosporangio de P. variabilis. 


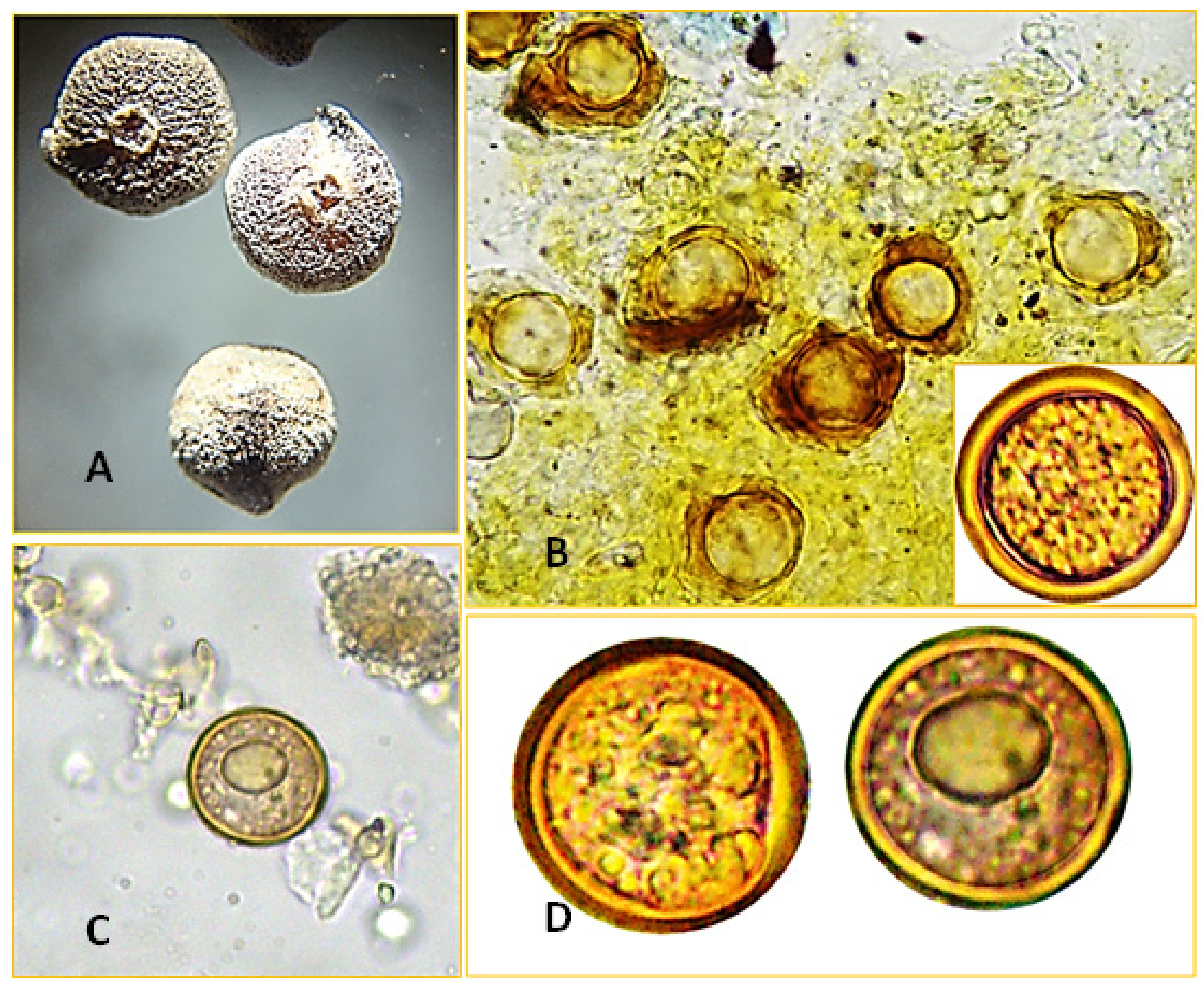

Figura 2. Oosporas de $P$. variabilis en quinua Pasankalla. A. grano de quinua antes del lavado (100X); B. sobre la hoja de quinua (hojas traídas de Ayacucho) observadas a 400X; C y D. después del centrifugado y resuspensión para su recuento.

\section{Análisis del Área Bajo la Curva del Progreso de la Enfermedad (ABCPE)}

Hasta la 13ra evaluación, 54 días después de la siembra (dds) momento que coincidió con la última aplicación de los productos, los tratamientos metalaxyl (TM), azúcar fosfatado (KO) y fosfito de potasio (FP) presentaron los porcentajes más bajo de severidad con $0,71 \% ; 2,9 \%$ y $2,4 \%$ respectivamente, comparado con el testigo absoluto que tuvo $6,3 \%$ de severidad. A los 74 dds en la $20^{\mathrm{ma}}$ evaluación el testigo absoluto alcanzó $24,8 \%$ de severidad comparado con el tratamiento TM que obtuvo $15,8 \%$ (Fig. 3)

El tratamiento testigo absoluto sin aplicación (T) presentó el valor más alto del ABCPE con 442,43, seguido de los tratamientos Lactobacillus (E) y Fermentados de sólidos solubles (A) con 355,83 y 350,47 respectivamente sin mostrar diferencia estadística. De igual manera, los tratamientos azúcar fosfatado (KO), Azúcar fosfatado + Lactobacillus $(\mathrm{KO}+\mathrm{E})$, Fosfito de potasio + Fermentado de sólidos solubles (FP+A), Azúcar fosfatado +
Fermentados de sólidos solubles $(\mathrm{KO}+\mathrm{A})$, Fosfito de potasio + Lactobacillus (FP+E) y Fosfito de potasio (FP) no mostraron diferencias estadísticas con valores de 278,$82 ; 236,24 ; 229,72 ; 217,7 ; 204,27$ y 199,96 respectivamente. No obstante, el testigo Metalaxyl (TM) tuvo el valor más bajo del ABCPE con 175,43 (Fig.4, Tabla 2) indicando que existe control de mildiú en quinua Pasankalla en condiciones de la Molina, para siembra de la campaña 2013.

Coincidiendo con el reporte de Méndez (2010), para el control de mildiú de melón. El resultado de ABCPE para el fosfito fue significativo con respecto a otros inductores (Menadiona Bisulfito Sodio, AcibenzolarS-metil) que fueron similares al testigo. En relación al Metalaxyl, Gabriel et al. (2013), para el control de mildiú en diferentes variedades de quinua, aplicó metalaxyl en combinación con cimoxanil obteniendo los valores más bajos del ABCPE. 


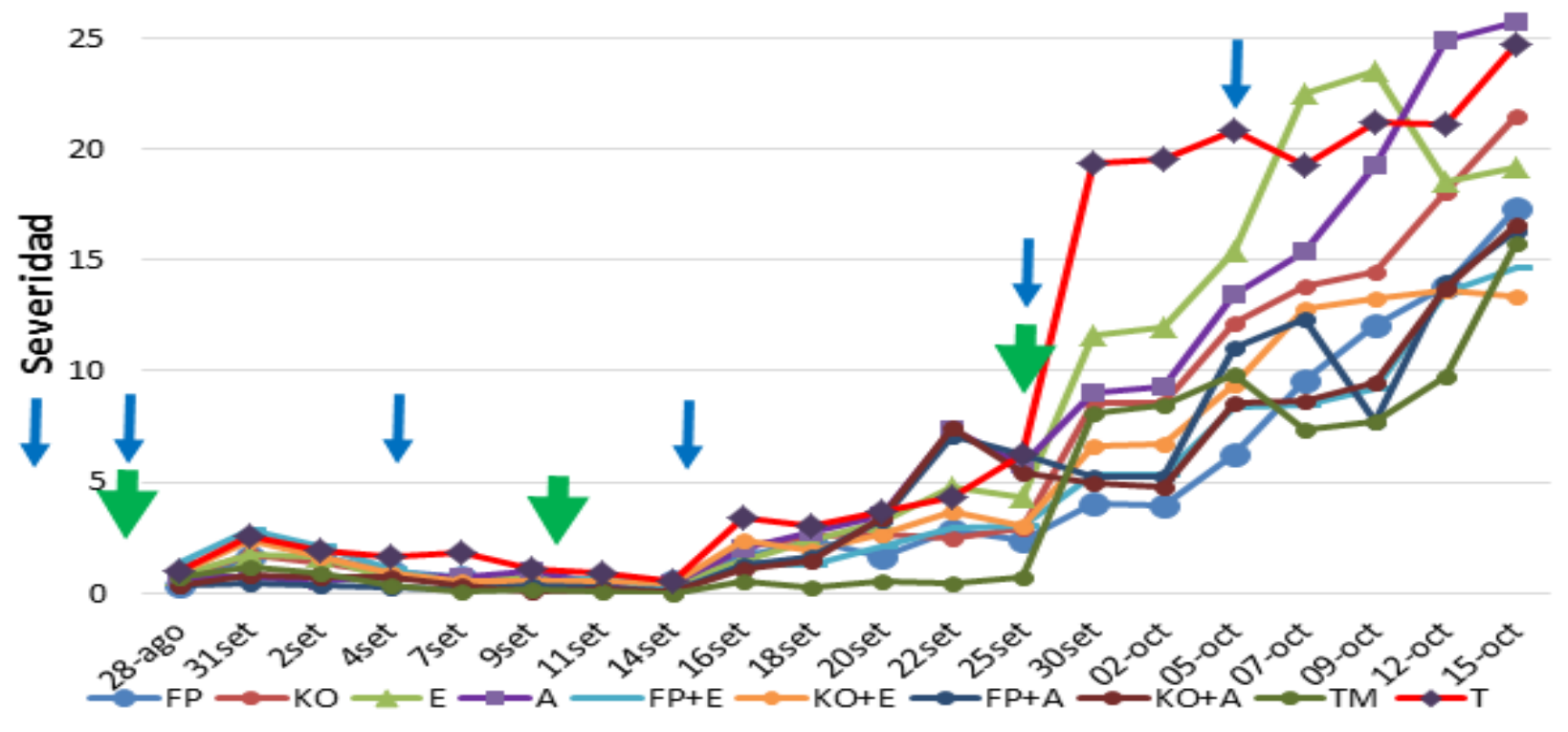

\begin{tabular}{llll}
\hline FP & : Fosfito de potasio & FP+E & : Fosfito de potasio + Lactobacillus \\
KO & : Azúcar fosfatado & KO+E & : Azúcar fosfatado + Lactobacillus \\
E & $:$ Lactobacillus (Ecovida) & FP+A & : Fosfito de potasio + Fermentados de sólidos solubles \\
A & : Fermentados de sólidos solubles & KO+A & : Azúcar fosfatado + Fermentados de sólidos solubles \\
TM & : Testigo Metalaxyl & T & : Testigo absoluto (sin aplicación) \\
\hline
\end{tabular}

Figura 3. Curvas del progreso de las diferentes epidemias como respuesta a los tratamientos para el control de $P$. variabilis. La Molina 2013.

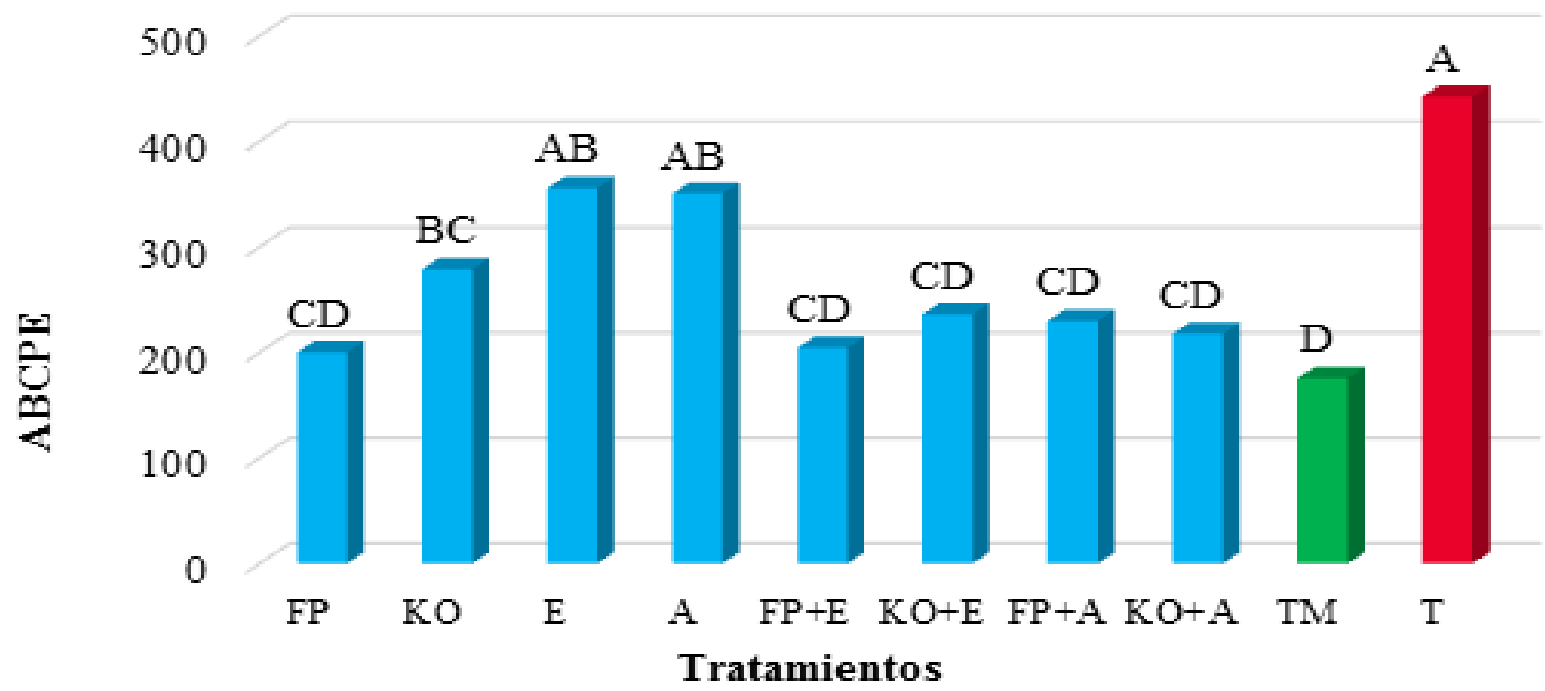

\begin{tabular}{|c|c|c|c|}
\hline$\overline{\mathrm{FP}}$ & : Fosfito de potasio & $\mathrm{FP}+\mathrm{E}$ & Fosfito de potasio + Lactobacillus \\
\hline $\mathrm{KO}$ & : Azúcar fosfatado & $\mathrm{KO}+\mathrm{E}$ & : Azúcar fosfatado + Lactobacillus \\
\hline $\mathrm{E}$ & : Lactobacillus (Ecovida) & $\mathrm{FP}+\mathrm{A}$ & Fosfito de potasio + Fermentados de sólidos solubles \\
\hline A & : Fermentados de sólidos solubles & $\mathrm{KO}+\mathrm{A}$ & : Azúcar fosfatado + Fermentados de sólidos solubles \\
\hline TM & : Testigo Metalaxyl & $\mathrm{T}$ & : Testigo absoluto (sin aplicación) \\
\hline
\end{tabular}


Desde el inicio de la evaluación de severidad del mildiú (28 de agosto) y la última evaluación (15 de octubre) las condiciones de temperatura oscilaron entre 15 y $17{ }^{\circ} \mathrm{C}$ y la humedad relativa entre 85 y $95 \%$, indicando que hubo condiciones óptimas para el desarrollo del mildiú. Sin embargo, en la Fig. 3, observamos que a partir del 25 de septiembre (13 vo evaluación) cuando el cultivo tuvo 54 dds las curvas del progreso de la enfermedad de los tratamientos se incrementaron a una mayor tasa, León (2003), indica que entre los 55 y 60 días de la siembra ocurre la fase de panojamiento en quinua produciéndose la emergencia del ápice de la planta, aglomeración de hojas pequeñas las cuales van cubriendo la panoja en sus tres cuartas partes, así mismo amarillamiento del primer par de hojas verdaderas (hojas que ya no son fotosintéticamente activas) y se producen una fuerte elongación del tallo y engrosamiento. De acuerdo a esta aseveración podemos presumir que el debilitamiento de los tejidos por la elongación en este periodo hace más susceptibles a los patógenos, por ello se incrementó la severidad de la enfermedad en esta etapa de inicio de panojamiento. Así mismo, Kumar et al. (2006), hizo el primer reporte de mildiú de la quinua en la India y observó que el pico más alto de severidad (ABCPE) se dio a los 65 días y posteriormente decreció.

\section{Comparación de la tasa a través del valor rho}

El mildiú, al ser una enfermedad policíclica es influenciado por factores ambientales, resistencia del cultivo y las aplicaciones de productos para su control; esta enfermedad influye en la tasa y cantidad de esporulación en una misma estación de crecimiento (Agrios 1995). La tasa, es otro parámetro para medir el efecto de las aplicaciones y comparar estadísticamente el efecto de los tratamientos (Madden 1986). La tasa, del progreso de la enfermedad ( $r$ ) de los tratamientos (epidemias) en esta investigación no pudieron ser comparados directamente ya que los tratamiento se ajustaron a diferentes modelos epidemiológicos (Exponencial, Logístico y Gómpertz). Por ello, se estandarizaron con la tasa relativa de incremento de la enfermedad ( $r h o$ ). Los valores obtenidos fueron sometidos a una comparación de medias Tukey (Fig. 5, Tabla 2), donde, el tratamiento fermentados solubles (A) mostró el valor rho más alto con 0,00086 (r $=0,027$ ), e indicó que la epidemia tuvo una tasa superior en comparación al resto de los tratamientos. En contraste, el tratamiento Fosfito de potasio + Lactobacillus (FP+E) resultó con la tasa relativa ( $r h o$ ) más baja de $0,00050(\mathrm{r}$ $=0,015)$, seguido de los tratamientos Fosfito de potasio (FP) y Metalaxyl (TM) con valor rho igual a 0,00056 $(\mathrm{r}=0,017)$ indicando que fueron los tratamientos que tuvieron mayor influencia en la reducción de la tasa de desarrollo del mildiú en quinua Pasankalla. A su vez, se encontró relación directa entre los valores de la tasa relativa y el ABCPE (Tabla 2) corroborando con ambas variables el control de la enfermedad con la aplicación de estos productos.

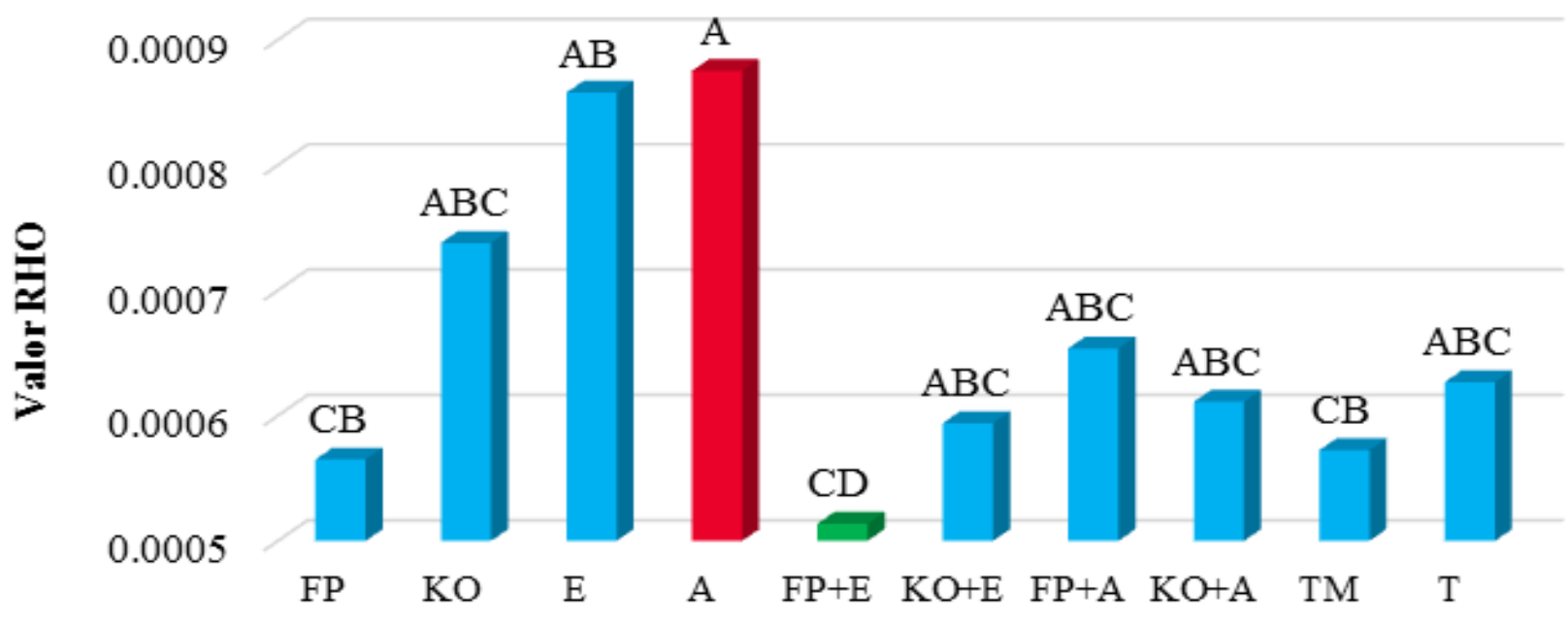

Tratamientos

\begin{tabular}{llll}
\hline FP & : Fosfito de potasio & FP+E & $:$ Fosfito de potasio + Lactobacillus \\
KO & $:$ Azúcar fosfatado & KO+E & $:$ Azúcar fosfatado + Lactobacillus \\
E & $:$ Lactobacillus (Ecovida) & FP+A & $:$ Fosfito de potasio + Fermentados de sólidos solubles \\
A & $:$ Fermentados de sólidos solubles & KO+A & $:$ Azúcar fosfatado + Fermentados de sólidos solubles \\
TM & $:$ Testigo Metalaxyl & T & $:$ Testigo absoluto (sin aplicación) \\
\hline
\end{tabular}

Figura 5. Comparación de las medias Tukey para tasa relativa del progreso de la enfermedad (rho). Nivel de significancia de 0,05. La Molina 2013. 


\section{Cuantificación de Oosporas}

Mercado (2001), encontró infecciones del perigonio y del pericarpio de quinua con oosporas provenientes de diferentes regiones del Perú, Así mismo, Testen (s.f.), a través de pruebas moleculares, también encontró oosporas de P. variabilis en lotes comerciales de diferentes países, entre ellos Perú. Demostrándose en ambos casos que, existe transmisión de $P$. variabilis a través de semilla botánica. En esta investigación, se detectó oosporas en semilla de quinua Pasankalla y se confirmó su trasmisión por semilla. Se contabilizó y se observó que el número de oosporas varió de acuerdo a los tratamientos (Fig. 6, Tabla 2). El testigo absoluto $(\mathrm{T})$ presentó 17500 , el tratamiento azúcar fosfatado + fermentados de sólidos solubles (KO+A) 16000 y azúcar fosfatado (KO) 14500 oosporas/ $\mathrm{kg}$ de semilla de quinua. Estos fueron estadísticamente iguales y diferentes a los tratamientos $\mathrm{FP}+\mathrm{E}, \mathrm{KO}+\mathrm{E}, \mathrm{FP}$, $\mathrm{E}, \mathrm{FP}+\mathrm{A}$ y $\mathrm{TM}$ y, a su vez, estos últimos no mostraron diferencias estadísticas. Cabe indicar que, el valor más bajo para el número de oosporas fue de 3500 , encontrados en los tratamientos fosfito de potasio + fermentados de sólidos solubles $(\mathrm{FP}+\mathrm{A})$ y metalaxyl (TM).

\section{Rendimiento}

El tratamiento FP mostró un resultado superior con 4059,70 kg/ha, seguido de los tratamientos Metalaxyl (TM), Fosfito de potasio + Lactobacillus (FP+E), Azúcar fosfatado (KO) y Azúcar fosfatado + Lactobacillus $(\mathrm{KO}+\mathrm{E})$ con 3686,$4 ; 3568,8 ; 3498.7$ y $3496,6 \mathrm{~kg} /$ ha respectivamente $\sin$ diferencias estadísticas. Los tratamientos con Fosfito de potasio + Fermentados de sólidos solubles (FP+A), Fermentados de sólidos solubles (A), Lactobacillus (E), Testigo absoluto (T) y Azúcares fosfatados + Fermentados de sólidos solubles $(\mathrm{KO}+\mathrm{A})$ fueron los que mostraron menor rendimientos con 3266,$2 ; 3184,7 ; 3184,7 ; 3131,6$ y 3077,9 respectivamente

(Fig. 7).

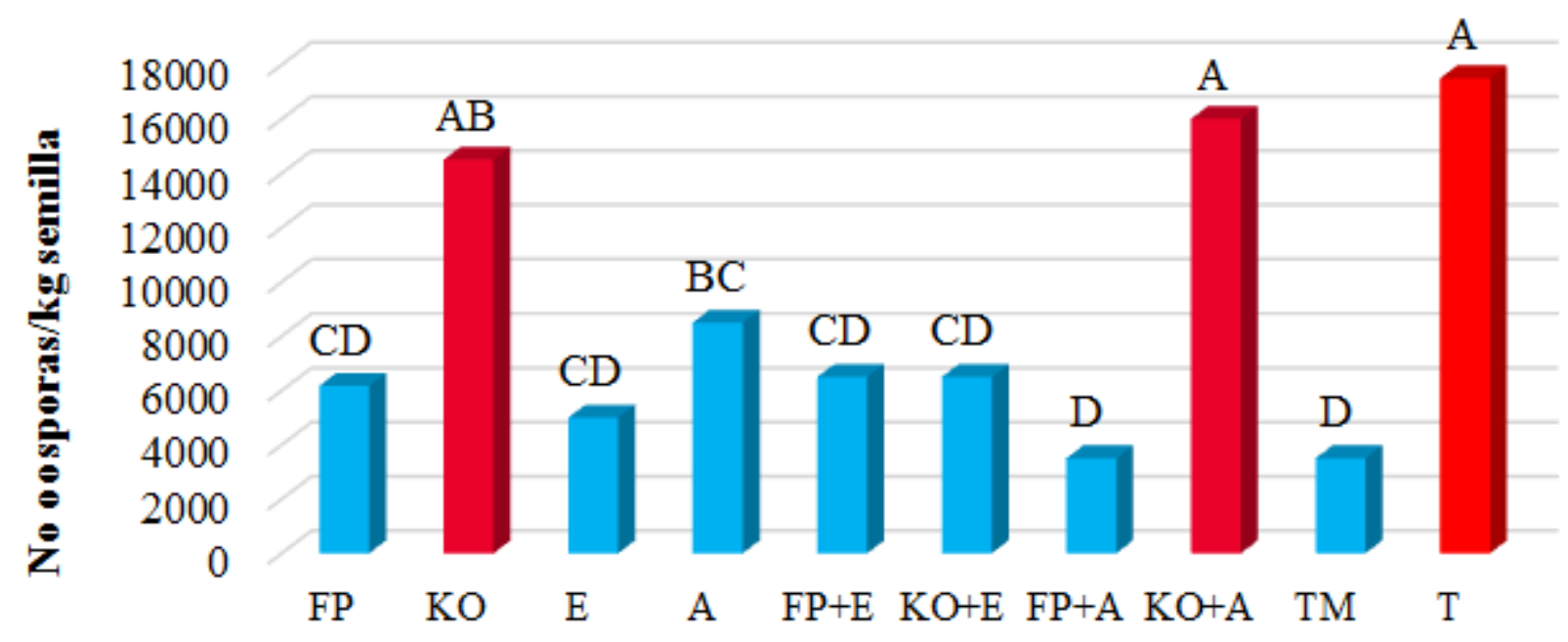

Tratamientos

\begin{tabular}{llll}
\hline FP & : Fosfito de potasio & FP+E & $:$ Fosfito de potasio + Lactobacillus \\
KO & : Azúcar fosfatado & KO+E & $:$ Azúcar fosfatado + Lactobacillus \\
E & : Lactobacillus (Ecovida) & FP+A & $:$ Fosfito de potasio + Fermentados de sólidos solubles \\
$\mathrm{A}$ & : Fermentados de sólidos solubles & $\mathrm{KO}+\mathrm{A}$ & $:$ Azúcar fosfatado + Fermentados de sólidos solubles \\
$\mathrm{TM}$ & $:$ Testigo Metalaxyl & $\mathrm{T}$ & $:$ Testigo absoluto (sin aplicación) \\
\hline
\end{tabular}

Figura 6: Comparación de las medias Tukey para el número de oosporas por kg de semilla. Nivel de significancia de 0,05. La Molina 2013.

Los productos con fosfito de potasio generan las defensas naturales y controlan de manera muy efectiva al mildiú y Phoma lo cual permite obtener altos rendimientos en el cultivo de quinua (Pozo, 2014). El producto Kalex es un fertilizante especial a base de fosfito de potasio, cuya actividad está ligada a la actividad del ión $\left(\mathrm{PO}_{3}^{-}\right)$que, por su elevada sistemicidad y desarrollo de ácido fosforoso, induce a la planta a la producción de fitoalexinas predisponiendo a las plantas a autodefenderse contra las enfermedades. Este producto ayuda a la eficacia nutritiva del fósforo y del potasio (Química Suiza 2013).
El rol del potasio en la resistencia a enfermedades fue ampliamente reportado, puesto que hay una relación inversa entre suelos con potasio disponible y la severidad de enfermedades (Huber y Arny 1985). En trabajos donde se hicieron aplicaciones de potasio resultaron en la supresión de enfermedades foliares (Harris 1997) y fertilizantes de potasio aplicados foliarmente fueron inductores altamente efectivos en la protección sistémica contra enfermedades, entre ellas el oídium (Reuveni et al 1995), además, el potasio está involucrado en numerosas funciones fisiológicas relacionadas a la tolerancia al estrés 
biótico y abiótico (Oosterhuis 2014). En forma general, la nutrición mineral afecta la susceptibilidad de las plantas a enfermedades especialmente cuando hay deficiencia. Tanto macro y micro elementos son esenciales en varios mecanismos como: la activación de enzimas que produce metabolitos de defensa así como ligninas y fitoalexinas (Elmer 2014). En esta investigación el tratamiento con fosfito de potasio tiene las características de fertilizante foliar con contenidos de fósforo y potasio, los cuales influyeron en la activación de resistencia contra el mildiú y al estrés que generó la infección.

El metalaxyl, pertenece al grupo de la Phenilamidas, inhibe la síntesis de ácidos nucleicos y tiene buen control sobre Oomycetes. Sin embargo, tiene un alto riesgo de resistencia cruzada con usos frecuentes (FRAC 2010). Aislamientos de Phytophthora frente a las Phenilamidas, gradualmente, vienen siendo una parte importante de poblaciones resistentes en muchos países. No obstante, las mezclas que contienen phenilamidas siguen siendo una estrategia efectiva y exitosa para su control, ante los continuos cambios en poblaciones del mildiú se requiere una cuidadosa adaptación de los programas de control de la enfermedad (Gisi 1996). Saharan (1992), Verman et al. (1994), demostraron buena eficacia en el control de mildiú en diferentes cultivos, empleando fungicidas, entre ellos el Metalaxyl. Así mismo, Saharan et al. (1997), con tres aplicaciones foliares de Metalaxyl y mancozeb en el cultivo de mostaza a intervalos de 20 días, 40 días después de la siembra, lograron controlar hasta $82 \%$ de mildiú incrementando el rendimiento en 49 $\%$. Xiaojun (2013), reporta altos niveles de resistencia a metalaxyl en poblaciones de mildiú del melón causado por Psedoperonospora cubensis, en la provincia de Shanxi en China. Por su parte, Couso (2013), reportó que, en Galicia- España en áreas de siembra de papa donde el suelo se maneja en forma intensiva por más de 50 años, presencia de residuos de metalaxyl en aguas superficiales de drenaje en épocas de poca lluvias. Estos trabajos indican que el metalaxyl es una buena alternativa para el control de Oomycetes pero su uso excesivo genera resistencia y contamina el medioambiente. Por ello, se buscó alternativas para el manejo del mildiú en quinua.

\section{Calidad}

De acuerdo a la comparación de medias Tukey con un nivel de significación de 0,05 no hubo diferencia estadística entre los tratamientos para el porcentaje de proteína, peso de 1000 granos y granulometría.

\section{Análisis económico}

El tratamiento a base de Fosfito de potasio (FP) generó $37,05 \%$ más de ingreso, sobre las ganancias del testigo absoluto seguido de los tratamientos Metalaxyl (TM) con 21,45\% y Fosfito de potasio + Lactobacillus (FP+E) con $16,16 \%$. No obstante, los tratamientos Lactobacillus (E), Azúcar fosfatado + Fermentados de sólidos solubles $(\mathrm{KO}+\mathrm{A})$ registraron pérdidas (Tabla 3 ).

Tabla 2. Comparación de medias Tukey para el ABCPE, tasa del progreso del mildiú ( $r)$, número de oosporas $(\mathrm{N} \% / \mathrm{kg})$ y rendimiento (kg/ha). Nivel de significancia de 0,05. La Molina 2013.

\begin{tabular}{|c|c|c|c|c|c|c|c|c|c|}
\hline \multirow{2}{*}{$\frac{\text { Código }}{\text { FP }}$} & \multicolumn{2}{|c|}{ ABCPE } & \multirow{2}{*}{$\frac{\mathrm{r}}{0.018}$} & \multicolumn{2}{|r|}{ Rho } & \multicolumn{2}{|c|}{$\begin{array}{l}\text { Oospora/kg de } \\
\text { semilla }\end{array}$} & \multicolumn{2}{|c|}{ Rendimiento $(\mathrm{kg} / \mathrm{ha})$} \\
\hline & 200.0 & $\mathrm{CD}$ & & 0.00056 & $\mathrm{CB}$ & 6167 & $\mathrm{CD}$ & 4059.7 & A \\
\hline KO & 278.8 & $\mathrm{BC}$ & 0.023 & 0.00073 & $\mathrm{ABC}$ & 14500 & $\mathrm{AB}$ & 3498.7 & $\mathrm{AB}$ \\
\hline E & 355.8 & $\mathrm{AB}$ & 0.027 & 0.00085 & $\mathrm{AB}$ & 5000 & $\mathrm{CD}$ & 3184.7 & B \\
\hline A & 350.5 & $\mathrm{AB}$ & 0.027 & 0.00087 & A & 8500 & $\mathrm{BC}$ & 3184.7 & B \\
\hline $\mathrm{FP}+\mathrm{E}$ & 204.3 & $\mathrm{CD}$ & 0.016 & 0.00050 & $\mathrm{C}$ & 6500 & $\mathrm{CD}$ & 3568.8 & $\mathrm{AB}$ \\
\hline $\mathrm{KO}+\mathrm{E}$ & 236.2 & $\mathrm{CD}$ & 0.018 & 0.00058 & $\mathrm{ABC}$ & 6500 & $\mathrm{CD}$ & 3496.6 & $\mathrm{AB}$ \\
\hline $\mathrm{FP}+\mathrm{A}$ & 229.8 & $\mathrm{CD}$ & 0.020 & 0.00064 & $\mathrm{ABC}$ & 3500 & $\mathrm{D}$ & 3266.2 & B \\
\hline $\mathrm{KO}+\mathrm{A}$ & 217.7 & $\mathrm{CD}$ & 0.019 & 0.00060 & $\mathrm{ABC}$ & 16000 & A & 3077.9 & B \\
\hline $\mathrm{TM}$ & 175.4 & $\mathrm{D}$ & 0.018 & 0.00056 & $\mathrm{CB}$ & 3500 & $\mathrm{D}$ & 3686.4 & $\mathrm{AB}$ \\
\hline $\mathrm{T}$ & 442.4 & A & 0.019 & 0.00062 & $\mathrm{ABC}$ & 17500 & A & 3131.6 & \\
\hline \multicolumn{4}{|c|}{ Fosfito de potasio } & $\mathrm{FP}+\mathrm{E}$ & \multicolumn{5}{|c|}{ : Fosfito de potasio + Lactobacillus } \\
\hline : Azi & \multicolumn{3}{|c|}{ : Azúcar fosfatado } & $\mathrm{KO}+\mathrm{E}$ & \multicolumn{5}{|c|}{ : Azúcar fosfatado + Lactobacillus } \\
\hline$: \mathrm{Lac}$ & \multicolumn{3}{|c|}{ : Lactobacillus } & $\mathrm{FP}+\mathrm{A}$ & \multicolumn{5}{|c|}{ : Fosfito de potasio + Fermentados de sólidos solubles } \\
\hline : Fer & \multicolumn{3}{|c|}{ : Fermentados de sólidos solubles } & $\mathrm{KO}+\mathrm{A}$ & \multicolumn{5}{|c|}{$\begin{array}{l}\text { : Fosfito de potasio + Fermentados de sólidos solubles } \\
: \text { Azúcar fosfatado + Fermentados de sólidos solubles }\end{array}$} \\
\hline TM : Tes & \multicolumn{3}{|c|}{ : Testigo Metalaxyl } & $\mathrm{T}$ & \multicolumn{5}{|c|}{ Testigo absoluto (sin aplicación) } \\
\hline
\end{tabular}




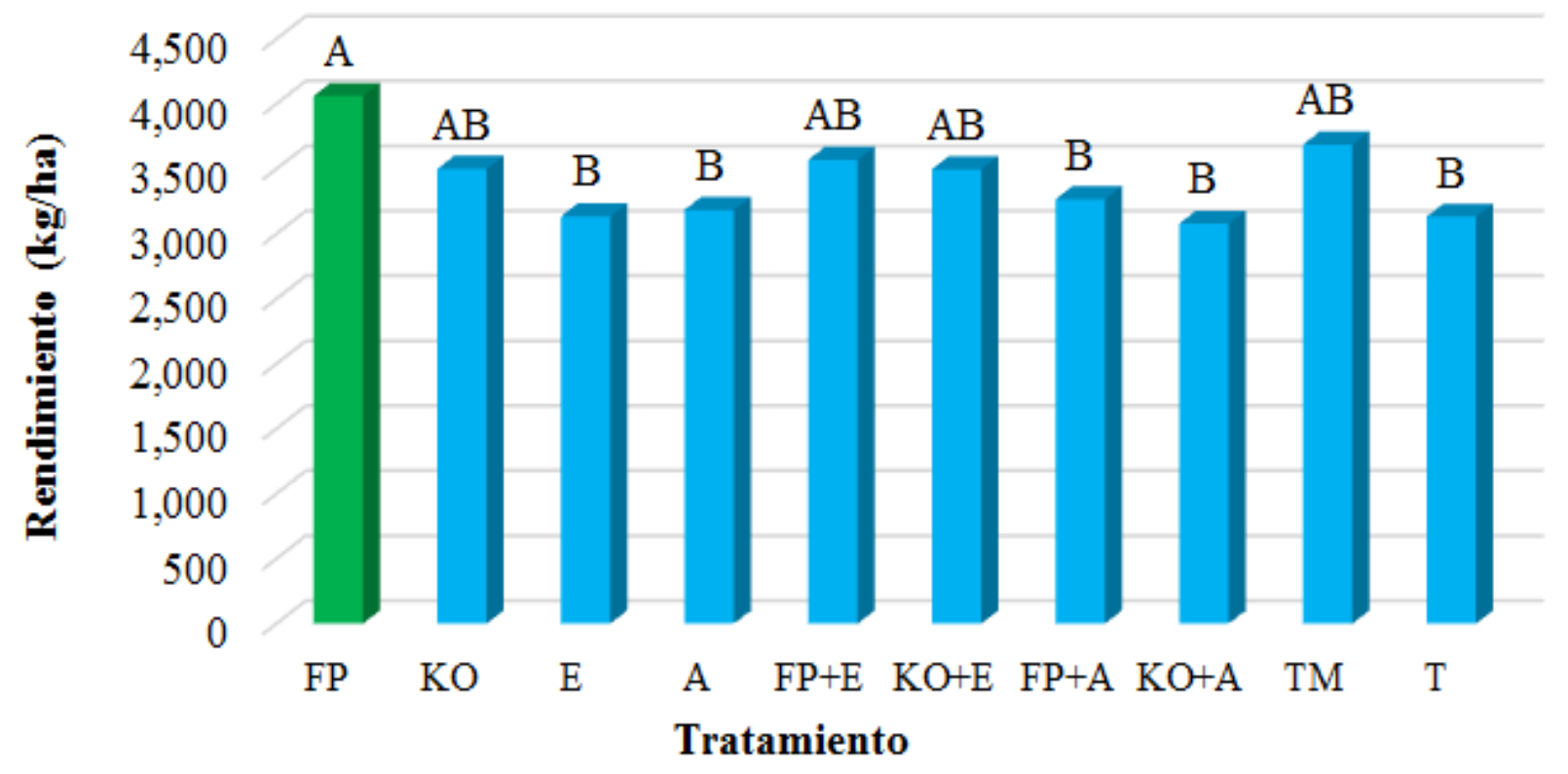

\begin{tabular}{llll}
\hline FP & : Fosfito de potasio & FP+E & : Fosfito de potasio + Lactobacillus \\
KO & : Azúcar fosfatado & KO+E & : Azúcar fosfatado + Lactobacillus \\
$\mathrm{E}$ & : Lactobacillus (Ecovida) & $\mathrm{FP}+\mathrm{A}$ & $:$ Fosfito de potasio + Fermentados de sólidos solubles \\
$\mathrm{A}$ & : Fermentados de sólidos solubles & $\mathrm{KO}+\mathrm{A}$ & $:$ Azúcar fosfatado + Fermentados de sólidos solubles \\
$\mathrm{TM}$ & : Testigo Metalaxyl & $\mathrm{T}$ & $:$ Testigo absoluto (sin aplicación) \\
\hline
\end{tabular}

Figura 7: Comparación de las medias Tukey para el rendimiento. Nivel de significancia de 0,05. La Molina 2013.

Tabla 3. Análisis económico de la producción de una hectárea de quinua Pasankalla con diferentes tratamientos para el control del mildiú en condiciones de La Molina 2013.

\begin{tabular}{|c|c|c|c|c|c|c|c|}
\hline \multicolumn{2}{|c|}{ Tratamiento } & $\begin{array}{c}\text { Costo del } \\
\text { producto }(\mathrm{S} / .) / \text { ha }\end{array}$ & $\begin{array}{l}\text { Costo total * } \\
(\mathrm{S} / .) / \mathrm{ha}\end{array}$ & $\begin{array}{l}\text { Rendimiento }(\mathrm{kg} / \\
\mathrm{ha})\end{array}$ & Ingreso** $(\mathrm{S} /)$. & $\begin{array}{l}\text { Utilidad por } \\
\text { ha }(\mathrm{S} / .)\end{array}$ & $\begin{array}{l}\text { Incremento de } \\
\text { utilidad }(\%)\end{array}$ \\
\hline \multicolumn{2}{|l|}{$\overline{\mathrm{FP}}$} & 130 & $6,742.67$ & 4059.69 & $40,596.88$ & $33,854.20$ & 37.05 \\
\hline \multicolumn{2}{|l|}{$\mathrm{KO}$} & 460 & $7,072.67$ & 3498.69 & $34,986.88$ & $27,914.20$ & 13.00 \\
\hline \multicolumn{2}{|l|}{$\mathrm{E}$} & 250 & $6,862.67$ & 3133.63 & $31,336.25$ & $24,473.58$ & -0.93 \\
\hline \multicolumn{2}{|l|}{ A } & 525 & $7,137.67$ & 3184.73 & $31,847.29$ & $24,709.62$ & 0.03 \\
\hline \multicolumn{2}{|l|}{$\mathrm{FP}+\mathrm{E}$} & 380 & $6,992.67$ & 3568.81 & $35,688.13$ & $28,695.45$ & 16.16 \\
\hline \multicolumn{2}{|c|}{$\mathrm{KO}+\mathrm{E}$} & 710 & $7,322.67$ & 3496.63 & $34,966.25$ & $27,643.58$ & 11.90 \\
\hline \multicolumn{2}{|c|}{$\mathrm{FP}+\mathrm{A}$} & 655 & $7,267.67$ & 3266.24 & $32,662.40$ & $25,394.73$ & 2.80 \\
\hline \multicolumn{2}{|c|}{$\mathrm{KO}+\mathrm{A}$} & 985 & $7,597.67$ & 3077.94 & $30,779.38$ & $23,181.70$ & -6.16 \\
\hline \multicolumn{2}{|c|}{ TM } & 250 & $6,862.67$ & 3686.38 & $36,863.75$ & $30,001.08$ & 21.45 \\
\hline \multicolumn{2}{|l|}{$\mathrm{T}$} & 0 & $6,612.67$ & 3131.56 & $31,315.63$ & $24,702.95$ & 0.00 \\
\hline \multirow{3}{*}{$\begin{array}{l}\mathrm{FP} \\
\mathrm{KO} \\
\mathrm{E}\end{array}$} & \multicolumn{2}{|c|}{ : Fosfito de potasio } & $\mathrm{FP}+\mathrm{E}$ & \multicolumn{4}{|c|}{ : Fosfito de potasio + Lactobacillus } \\
\hline & \multirow{4}{*}{\multicolumn{2}{|c|}{$\begin{array}{l}\text { : Azúcar fosfatado } \\
\text { : Lactobacillus } \\
\text { : Fermentados de sólidos } \\
\text { solubles } \\
\text { : Testigo Metalaxyl }\end{array}$}} & $\mathrm{KO}+\mathrm{E}$ & \multirow{2}{*}{\multicolumn{4}{|c|}{$\begin{array}{l}\text { : Azúcar fosfatado }+ \text { Lactobacillus } \\
: \text { Fosfito de potasio }+ \text { Fermentados de sólidos solubles }\end{array}$}} \\
\hline & & & $\mathrm{FP}+\mathrm{A}$ & & & & \\
\hline A & & & $\mathrm{KO}+\mathrm{A}$ & \multicolumn{4}{|c|}{ : Azúcar fosfatado + Fermentados de sólidos solubles } \\
\hline TM & & & $\mathrm{T}$ & : Testigo absoluto & (sin aplicación) & & \\
\hline
\end{tabular}

\section{Conclusiones}

Se evidenció infecciones por $P$. variabilis en quinua Pasankalla, en siembra de invierno en La Molina, campaña 2013. El testigo absoluto (T) mostró el valor más alto del $\mathrm{ABCPE}$; mientras que, los valores más bajos fueron obtenidos en los tratamientos Metalaxyl (TM) y Fosfito de potasio (FP). La tasa del progreso de la enfermedad $(r)$ más baja fue obtenida en los tratamientos Fosfito de potasio + Lactobacillus $(\mathrm{FP}+\mathrm{E})$, Fosfito de potasio (FP) y Metalaxyl (TM). El menor número de oosporas/kg de semilla se observó en los tratamientos Metalaxyl (TM) y Fosfito de potasio + Fermentados de solidos solubles (FP+A); entre tanto, el Testigo absoluto presentó el mayor número. Los tratamientos con Fosfito 
de potasio (FP), Metalaxyl (TM) y Fosfito de potasio + Lactobacillus (FP+E) tuvieron los mejores rendimientos. Los tratamientos fosfito de potasio (FP) y Metalaxyl (TM) generaron mayores ganancias con respecto al testigo absoluto (T).

\section{Literatura citada}

Agrios, G. N. 1995. Fitopatología Segunda edición. Departamento de Fitopatología de la Universidad de Massachusetts. p. 171

Couso, A. B.; Calviño, D. F.; Álvarez, M. A.; Gándara, J. S.; Nóvoa, J.C. y Estévez, M. A. 2013. Pollution of surface waters by metalaxyl and nitrate from non-point sources. Science of The Total Environment. Volumen 461-462, 1 September 2013, Pages 282-289

Danielsen, S. y Ames, T. 2008. El mildiú de la quinua en la zona andina (Peronospora farinosa) Manual práctico para el estudio de la enfermedad y del patógeno. (en línea) consultado el 14 de setiembre del 2011. Disponible en http://agris.fao.org/agris-search/search/ display.do? $\mathrm{f}=2008 /$.

Elmer, W. H. y Datnoff, L. E. 2014. Mineral Nutrition and Suppression of Plant Disease. Encyclopedia of Agriculture and Food Systems. Pages 231-244. (en línea) consultado el 01 de set. 2014. Disponible en http://www.sciencedirect.com/science/article/pii/ B9780444525123002515.

FRAC (Fungicide Resistance Action Committee). 2013. Fungicides sorted by mode of action. (en línea) consultado el 02 de set. 2014. Disponible en http:// www.frac.info/publication/anhang/FRAC $\% 20 \mathrm{Code} \% 20$ List\%202013-final.pdf.

Gabriel, J.; Luna, N.; Vargas, A.; Magne, J.; Angulo, A.; La Torre, J. y Bonifacio, A. 2013. Resistencia al mildiú (Peronospora variabilis) en variedades de quinua (Chenopodium quinoa Willd) en el valle bajo de Cochabamba.VI Congreso mundial de quinua. Ibarra, Ecuador. 36 diapositivas.

Gisi, U. y Cohen, Y. 1996. RESISTANCE TO PHENYLAMIDE FUNGICIDES: A Case Study withPhytophthora infestans Involving Mating Type and Race Structure. Annual Review of Phytopathology. 34: 549-572. Solo resumen. (en línea). Consultado el 02 de set. 2014. Disponible en http://www.annualreviews.org/ doi/abs/10.1146/annurev.phyto.34.1.549

Harris, G. 1997. Potassium deficiency in cotton linked to leafspot disease. Better Crops 81, 10-11. In: XVTH International Plant Protection Congress Beijin, China May 11-16, 2004.2 (en línea). Consultado 02 set. 2014. Disponible en http://www.iclfertilizers.com/Fertilizers/ Knowledge\%20Center/KCl_and_suppression of diseases.pdf.

Huber, D. M. y Arny, D. C. 1985. Interaction of potassium with plant disease. In: XVTH International Plant Protection Congress Beijin, China May 11-16, 2004.2 (en línea). Consultado 02 set. 2014. Disponible en
http://www.iclfertilizers.com/Fertilizers/Knowledge $\% 20$ Center $/ \mathrm{KCl}$ and suppression of diseases.pdff.

Kumar, A.; Bhargava, A.; Shukla, S.; Singh, H. B. y Ohri, D. 2006. Screening of exotic Chenopodium quinoa accessions for downy mildew resistance under mid-eastern conditions of India. Crop Protection 5 (2006) 879-889.

Madden, L. V. 1986. Statistical analysis and comparison of disease progress curves. Pg. 55-87 in Plant disease Epidemiology. Leonard, K. J. and W. E. Fry, eds. Macmillan. NY.

Mendez L., W.; Arauz, L. F. y Rios, R. 2010. Evaluación de fungicidas convencionales e inductores de resistencia para el combate de mildiú velloso (Pseudoperonospora cubensis) EN MELÓN (Cucumis melo). Agronomía Costarricense 34(2): 153-164. ISSN: 0377-9424.

Mercado L., V. H. 2001. El mildiú de la quinua y su transmisión por medio de semilla. Tesis para optar el título de ingeniero agrónomo. Universidad Nacional Agraria La Molina. Lima - Perú. 55 p.

MINAG - OEEE (Direcciones Regionales y Subregionales de Agricultura). 2014. superficie de siembra y cosechada. Base de datos. s.p.

Oosterhuis Derrick, M.; Loka Dimitra, A.; Kawakami Eduardo, M. y Pettigrew William, T. 2014. The Physiology of Potassium in Crop Production. Advances in Agronomy. Volume 126, 2014, Pages 203-233. Solo resumen.

Pozo C. 2014. Record nacional de rendimiento en quinua con el manejo fisionutricional (MFN). En Redagricola Perú No. 16. Julio/Agosto ISSN 0718-0802. Pg. 13.

Química Suiza. 2013. Ficha técnica de Kalex. (en línea). Lima, Perú. Consultado el 07 de mayo del 2014. Disponible en http://www.qsindustrial.biz/sites/default/ files/product/files/publics/ficha_tecnica_kalex.pdf.

Reuveni, M. 1995. Efficacy of foliar sprays of phosphonates in controlling powdery mildews in fieldgrownnectarine, mango tres and grapes. Crop Proteccion 14: 311-314

Saharan, G. S. 1992. Management of rapeseed and mustard disease. In: In: Thakur, R.P.; Mathur, K. 2001. Downy mildews of India. Crop Protection 21 (2002) 333-345.

Saharan, G. S.; Varma, P. R. y Nashaat, N. I. 1997. Monograph on Downy Mildew of Crucifers Saskatoon Research Center.Technical Bolletin, Agriculture and Agri-food Canada. In: Thakur, R.P.; Mathur, K. 2001. Downy mildews of India. Crop Protection 21 (2002) 333-345.

Shaner, G. y Finney, R. E. 1977. The effect of nitrogen fertilization on the expression of slow-mildewing resistance in knox wheat. Phytopathology 67: 1051-1056.

Testen, A.; Ochoa J.B.; Jovanna, P.; Jiménez, G. y M. M., Backman, P. s.f. Detección en semillas, filogenia y grupos de apareamiento de Peronospora variabilis en 
quinua. Implicaciones para el desarrollo de estrategias MIP. Department of Plant Pathology and Environmental Microbiology Pennsylvania State University. USA. 22 diapositivas.

Vanderplank, J. C. 1963. Plant diseases: epidemics and control. Academic Press, New York. 349 p.

Verma, P. R., Saharan, G. S. y Goyal, B. K. 1994. Peronospora parasítica (Pers. Ex Fr.) Fr (downy mildew) on crucifers. In: In: Thakur, R.P.; Mathur, K. 2001. Downy mildews of India. Crop Protection 21 (2002) 333-345.

Xiaojun, Z.; Lu, R.; Hui, Y.; Jianbo Z.; Jvcai, H. y Yong, L. 2013. Sensitivity of Pseudoperonospora cubensis to dimethomorph, metalaxyl and fosetyl-aluminium in Shanxi of China. Crop Protection. Volumen 43, Enero 2013, P. 38-44. 